\title{
Ein Beitrag zur Gliederung der eiszeitlichen Ablagerungen im Trauntal bei Wels/Oberösterreich ${ }^{1}$ )
}

Meinem verehrten Lehrer Prof. Dr. Hans Graul zu seinem 65. Geburtstag gewidmet

\author{
Von Kurt Metzger, Heidelberg
}

Mit 6 Abbildungen

$\mathrm{Z}$ us a m me $\mathrm{f}$ a s sung. Mit dem Beitrag soll gezeigt werden, daß es mit Hilfe geeigneter physikalisch-chemischer Untersuchungen im Labor und im Gelände möglich ist, über die Feldbefunde hinaus, Aussagen über Aufbau und Genese komplexer Profile zu machen. Der Lehmkomplex der Tongrube der Ziegelei Würzburger, Wels, kann nach den Untersuchungsergebnissen nicht als einheitliche Sedimentation aufgefaßt werden, er besteht vielmehr aus vier verschiedenen Akkumulationen. Damit ergibt sich für den liegenden Deckenschotter ein günzeiszeitliches Alter.

$\mathrm{Su} \mathrm{m} \mathrm{m}$ a r $\mathrm{y}$. This contribution should show that it is possible to give a more exact declaration with suitable chemical and physical experiments as investigations in the field alone. So one can give more exact declaration about structure and formation of complicated profiles. The claycomplex in the clay-pit of the brickworks Würzburger/Wels can not be accepted as an homogeneous sedimentition, it is rather a komplex of four accumulations. Therewith is the age of the lying "Deckenschotter" such of the glacial "Günz" period.

\section{A) Einleitung}

Angeregt durch Exkursionen mit den Herren KoHL (1968) und Janik (1970) im Gebiet der Traun-Enns-Platte entstand dieser Beitrag. Er soll zur Klärung unterschiedlicher Auffassungen über die Struktur der Deckschichtenprofile auf Deckschottern im Vorgebiet des

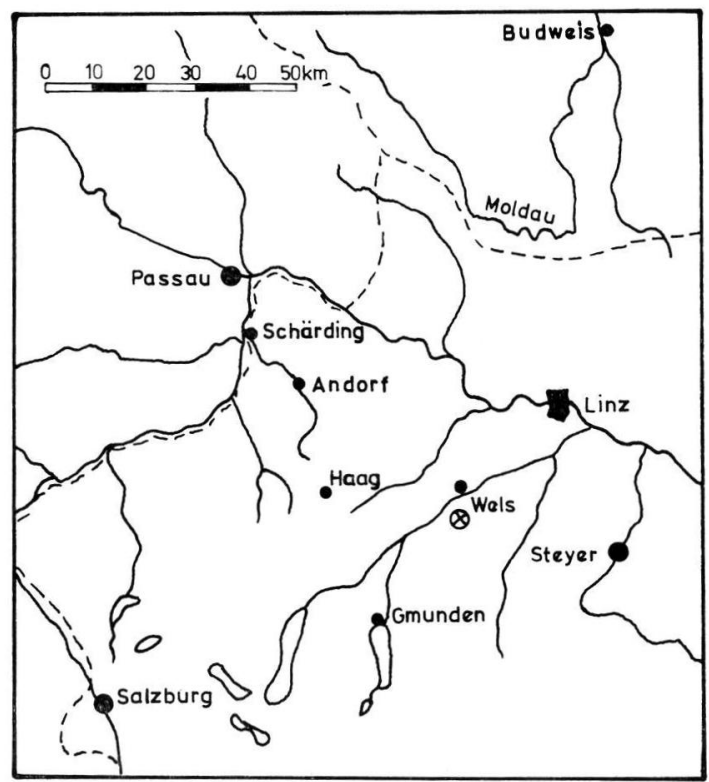

Abb. 1. Kartenskizze des Untersuchungsgebietes. Map stretch of the range of investigation. berg.

1) Aus dem Geomorphologischen Labor des Geographischen Instituts der Universität Heidel- 
ehemaligen Traungletschers dienen. Fink (1956) und KoHL (1955) beschreiben fossile Böden in den hangenden Feinsedimenten. JANIK (1965/67/69) widerspricht dieser Auffassung und behauptet, daß es sich hierbei um eine einheitliche Sedimentation handle, die durch keine warmzeitliche Bodenbildung unterbrochen sei. Er stützt sich dabei auf eingehende Laboruntersuchungen, wobei ein besonderes Schwergewicht auf der Interpretation von Bodendünnschliffen liegt. In der Staatsexamensarbeit von BAUER (1972) wird der Versuch unternommen, mit physikalisch-chemischen Methoden eine Entscheidung zugunsten der einen oder der anderen Hypothese herbeizuführen. Seine Untersuchungsergebnisse, sowie dazu ergänzende von mir durchgeführte Untersuchungen, werden im Folgenden einer eingehenden Betrachtung unterzogen. ${ }^{2}$ )

\section{B) Arbeitshypothese und Ergebnisse}

Durch geeignete physikalische und chemische Gelände- sowie Laboruntersuchungen soll geklärt werden, ob der Lehmkomplex (siehe Lageskizze Abb. 1) der Tongrube der Ziegelei Würzburger, Wels, durch einen „einheitlichen“ Sedimentationsprozeß entstanden ist.

1) Neben dem Feldbefund (siehe Aufschlußbeschreibung Abb. 2), der lediglich als Orientierungshilfe dienen soll, wurde ein Szintillationsdiagramm (Abb. 3) von der Aufschlußwand aufgenommen.

2) Aus dem Aufschluß wurden an markanten Stellen, die durch das Szintillationsdiagramm angezeigt sind, 12 Proben entnommen.

3) Als charakteristische Parameter, die sich bei Sedimentationsstillstand bzw. Abtrag des Sedimentationskörpers verändern, werden von mir nachfolgende Größen angesehen:

a) Gehalt an organischem Kohlenstoff ( $\mathrm{C}_{\text {org. }}$ )

b) Der Gehalt an kohlensaurem Calzium $\left(\mathrm{CO}_{2}\right)$ und anderen Erdkalielementen

c) Das $\mathrm{Fe}^{\mathrm{II}} / \mathrm{Fe}^{\mathrm{III}}$-Verhältnis

d) Der Retentionswert $\left(R_{f}\right.$-Wert)

e) Das Reflexionsvermögen (dieses objektive physikalische Verfahren ersetzt die weitgehend subjektive Farbwertsystematisierung mit Hilfe der Munsell-Skala)

f) Die Korngrößenzusammensetzung

g) Der Tonmineralbestand

h) Die Adsorptionsfähigkeit.

Von den acht angewandten Untersuchungsmethoden wurde beispielsweise die Bestimmung der Korngrößenzusammensetzung von JANIK ausführlich zur Beweisführung herangezogen. In diesem Zusammenhang interessiert, ob die Anwendung unterschiedlicher Methoden zu unterschiedlichen Ergebnissen führen kann. - JANIK arbeitet mit der Methode nach Kоретzкy (1914), wir hingegen mit der Köhn'schen Pipette.

\section{Beschreibung der Methoden und Erläuterung des Beweisgehaltes der Untersuchungen}

Zur Zeit der Begehung der Tongrube in Wels-Thalheim im August 1970 wurde an einer frischen Wand ca. $200 \mathrm{~m}$ Länge Lehm gewonnen. Der rezente Boden von ca. $40 \mathrm{~cm}$ Mächtigkeit war abgeschoben. Die Abbauwand zeigte eine scheinbar deutliche Schichtung, die durch die unterschiedlichen Braunwerte der Lehmlagen hervorgerufen wurde. Das Ergebnis einer näheren, eingehenden makroskopischen Untersuchung ist in der Aufschlußbe-

2) Die Arbeit wurde im Rahmen des DFG-Forschungsprogramms „Alpen im Eiszeitalter“ durchgeführt. Für die großzügige finanzielle und materielle Unterstützung möchte ich mich bedanken. 
schreibung unter Feldbefund wiedergegeben (Abb. 2). Es muß festgehalten werden, daß durch diese Untersuchung keinerlei Hinweis auf eine fossile Bodenbildung gefunden wurde. Die beschriebenen Eisen- und Mangankonkretionen sowie die Vergleyungshorizonte können keinesfalls als Zeugen einer pleistozänen Bodenbildung angesehen werden, ihr Vorhandensein kann ebensogut synsedimentären Prozessen zugeschrieben werden. Es ist im Folgenden zu prüfen, ob Laboruntersuchungen Aussagen in der einen oder anderen Richtung zulassen.

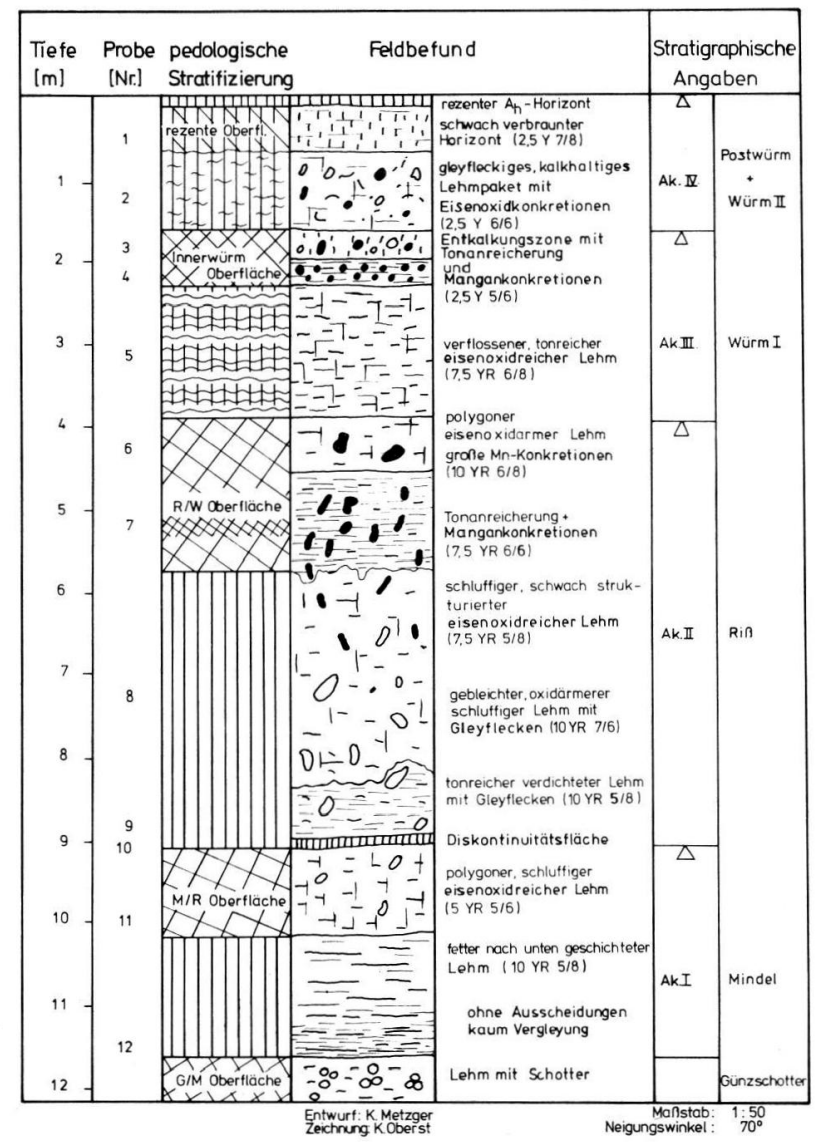

Abb. 2. Aufschlußbeschreibung mit pedologischer Stratifizierung und Datierung. Description of the clay-pit, pedological stratificing and dating.

1) Messungdernatürlichen Gamma-Aktivität an der A ufschluß w and

$\mathrm{Zu}$ dieser Untersuchung wurde eine Szintillationssonde, die in Zusammenarbeit mit der Fa. Gewerkschaft Brunhilde entwickelt wurde, benutzt. Die Auswertung der Meßkurve läßt Rückschlüsse auf Sedimentationsunterschiede sowie diagnetische Veränderungen im Sediment zu (Metzger 1970, Kunn 1971, Weinkötz 1972). Eine günstigere Meßgeometrie wurde durch eine ca. $10 \mathrm{~cm}$ tiefe Rille, die in die Aufschlußwand gegraben 
wurde, erhalten. Aus dem Diagramm (Abb. 3) geht hervor, daß der im Abbau befindliche Lehmkomplex keineswegs eine einheitliche Ablagerung darstellt, wie dies von JANIK angenommen wird. Bis zu einer Tiefe von $3 \mathrm{~m}$ zeigt die Kurve ein Sediment mit einem

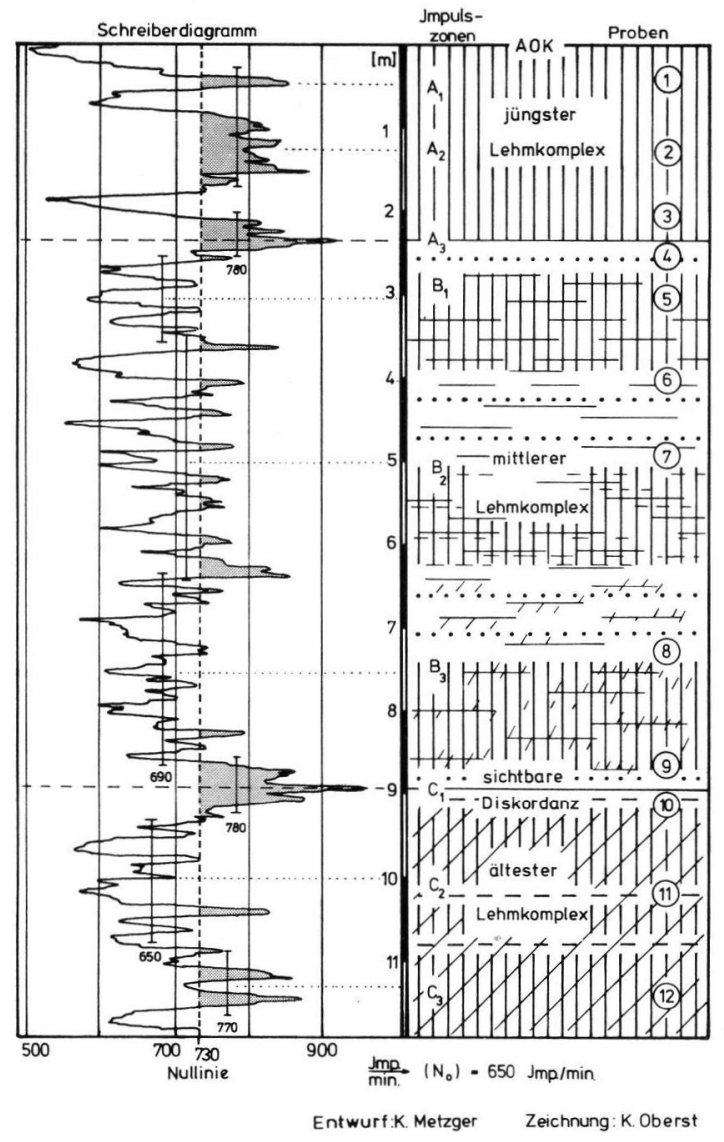

Abb. 3. Szintillationsdiagramm und seine Auswertung.

Diagram of the szintillation - counter and its interpretation.

Strahlungspegel über $730 \mathrm{Impulsen} / \mathrm{min}$. Im Gegensatz dazu liegt die natürliche Strahlung des Liegenden unter dem angegebenen Wert. Hier läßt die Gleichförmigkeit einen vom Hangenden unterschiedlichen zweiten Sedimentationsvorgang bis in eine Tiefe von $9 \mathrm{~m}$ AOK vermuten. Die in dieser Tiefe einsetzende starke Gamma-Intensität zeigt mit Sicherheit die Unterbrechung des Sedimentationsvorganges an. Die Interpretation der Strahlungskurve läßt zunächst eine Dreigliederung des anstehenden Lehmpaketes zu, wobei zwei Fragen offenbleiben:

a) werden durch das Abfallen der Gamma-Aktivität im Bereich des jüngsten Lehmkomplexes Sedimentationsintervalle angezeigt, oder handelt es sich bei dem Kurvenverlauf um eine rein statistische Erscheinung, die durch die Zerfallsimpulse des natürlichen Nulleffekts hervorgerufen wird?

b) Sprechen auch alle weiteren Meßwerte für die Geschlossenheit der drei durch die Gamma-Kurve ausgewiesenen Sedimentationskomplexe? 
Die eindeutige Antwort darauf löst die Frage nach der stratigraphischen Einordnung des Liegendschotters.

Argumente, die weiterführen, lassen sich u. a. durch Untersuchungen relevanter Bodenproben aus dem Profil finden. Dazu wird der Feldbefund mit dem aus der Gamma-Kurve interpretierten verglichen. An den Stellen, die abweichende Ergebnisse zeigen oder Übergänge vermuten lassen, werden Proben genommen. Ihnen stellt man Proben aus „zweifelsfreien" Zonen gegenüber. Diese Art der Untersuchung wird in Anlehnung an das Dualsystem gewählt, in dem die Antworten als „ja" oder als "nein" gewertet werden. Eine Anderung zur Vergleichsprobe wird demnach mit „ja“ gewertet und bestätigt oder widerlegt den Gamma-Kurvenverlauf. Eine detaillierte Interpretation der verschiedenen chemischen, physikalischen und mineralogischen Ergebnisse ist ohnehin erst sinnvoll, wenn sich durch die statistische Gesamtbetrachtung eine erkennbare Systematik in der Sedimentationsgenese abzeichnet. Um diesen Sachverhalt ganz deutlich zu sagen, die Analyse einzelner Parameter ist sehr reizvoll, und das sichtlich deswegen, weil das gewünschte Ergebnis erwartet werden darf. Im Folgenden sollen die Analysendaten zunächst nur auf ihren Verlauf in Bezug auf die Szintillationskurve untersucht werden.

\section{2) Chemische Untersuchungen an den Proben}

a) Gehalt an organischem Kohlenstoff

Der Gehalt an organischem Kohlenstoff (C org.) wird durch photometrische Bestimmung nach der nassen Oxidation mit Chromschwefelsäure (nach ANNes) ermittelt.

Mir erscheint der Gehalt an C org. im Probenmaterial als ein wichtiges Indiz für die Einheitlichkeit des Sedimentkörpers. Leider läßt seine Anhäufung nicht eindeutig auf eine ehemalige Oberfläche schließen. Denn C-Verbindungen organischer Natur können während der Sedimentation abgelagert werden und müssen nicht zwingend in situ in einem Boden entstehen. Auf der anderen Seite ist aber dann sicher, daß der organische Kohlenstoff in den untersuchten Lössen und Lößlehmen sein Vorhandensein einer mehr oder weniger entwickelten Pedosphäre im Einzugsgebiet der Sedimente verdankt. Auf die Kontamination des Sediments mit C org. würde die gleichmäßige Verteilung weit mehr allerdings eine Kohlenstoffanreicherung an der Basis der Sedimentschicht hinweisen. Denn zunächst wurde der kohlenstoffreiche Oberboden von der Erosion betroffen.

Der Vergleich des Verlaufs der organischen Kohlenstoffverteilung im Sediment - nach BAuer (1971) - mit der Gamma-log Kurve (Abb. 4) zeigt eine überraschend deutliche Übereinstimmung im Bereich $9 \mathrm{~m}$ unter AOK bei Probe 10. Unzweideutig ist ebenfalls die Aussage in $2 \mathrm{~m}$ Tiefe durch Probe 4, dagegen ist die Kohlenstoffanreicherung in $4 \mathrm{~m}$ Tiefe im Aktivitätsverlauf der Gammakurve nicht deutlich ausgeprägt. Jedenfalls unterstreicht die dreimalige Anhäufung des organischen Kohlenstoffs innerhalb des Sedimentationskörpers den Befund, den die Messung der natürlichen Gammaaktivität bringt. Einschränkend möchte ich sagen, daß an dieser Stelle noch immer nicht geklärt ist, ob Kohlenstoffanreicherung eine ehemalige Oberfläche andeutet oder den Beginn einer erneuten Sedimentationsphase. Für eine Stratifizierung im vorliegenden Fall wäre dieser Unterschied ohnehin nicht von Relevanz.

Bevor ich auf die Redoxverhältnisse in dem untersuchten Profil eingehe, soll in knappen Zügen die Verteilung von Karbonaten in dem Aufschluß beleuchtet werden.

b) Der Gehalt an kohlensaurem Calzium und anderer Erdkalielemente

Von verschiedenen Autoren (Mückenhausen 1959, Fezer 1969, u. a.) wird der Karbonatgehalt als Indikator für eine mehr oder weniger fortgeschrittene Verwitterung an- 


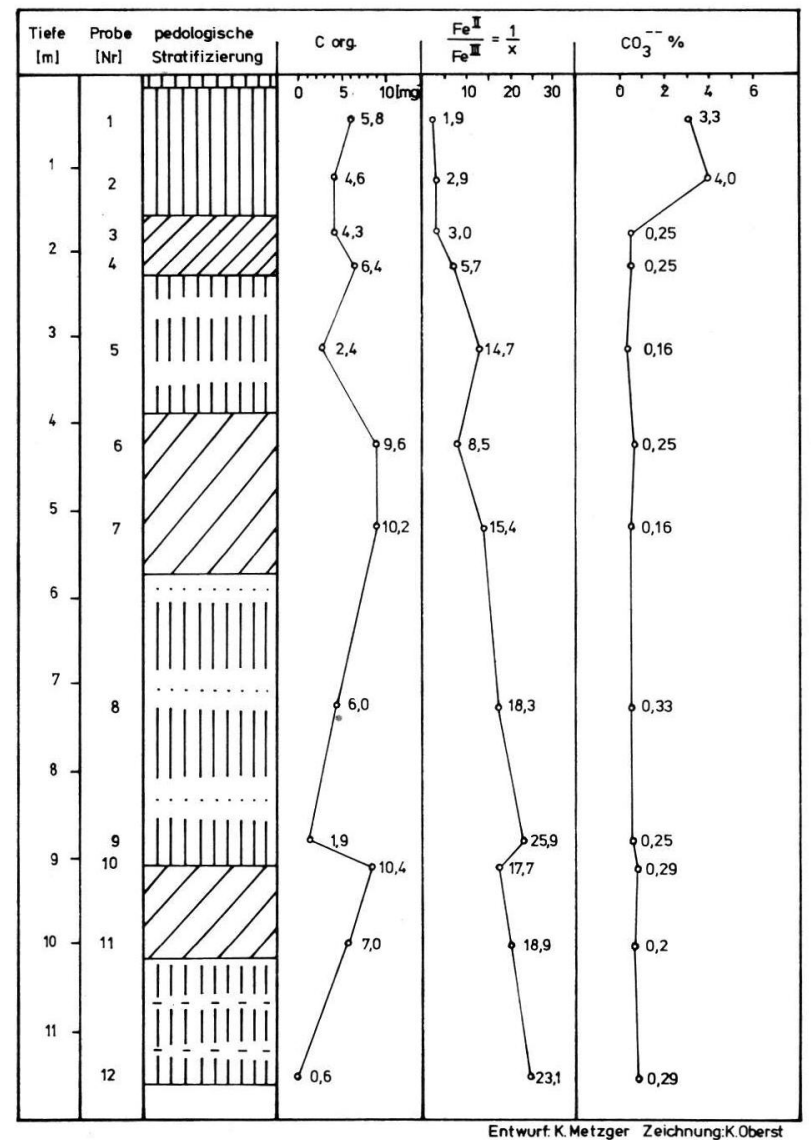

Abb. 4. Die aus chemischen Analysenwerten abgeleitete pedologische Stratifizierung. The pedological order found from chemical analysis data.

gesehen. Exakte Aussagen lassen sich hierbei sicher nur machen, wenn der ursprüngliche Gehalt an Karbonat bekannt ist. Bei den Sedimenten des Mittel- sowie Altpleistozän können nur Schätzungen einen Anhaltspunkt bringen. Diese Tatsache ändert aber nichts am Grundgehalt der Erkenntnis, daß eine längere Verwitterungsdauer zur Abnahme oder zur Neuverteilung des Karbonatgehalts im Sediment führt. Die mir vorliegenden Analysenwerte von BAUER (1971) und JANIK (1971) ${ }^{3}$ ) zeigen übereinstimmend die Karbonatgrenze bei $2 \mathrm{~m}$ unter AOK. Der Karbonatgehalt des Liegenden erreicht an keiner Stelle einen Wert von 0,5\% (Abb. 4). JANIK interpretiert den hohen Karbonatgehalt im Hangenden in dem o. a. Schreiben an mich folgendermaßen: „Beiliegend übersende ich Ihnen die Analysenergebnisse des Lehmprofils der Ziegelei Würzburger in Wels, wobei außer der Kalibestimmung nach der CAL-Methode noch das mit 1,67 n Ammoniumchloridlösung austauschbare Kali und die Kalifixierung des Bodens analysiert wurde. Dabei zeigt sich von oben nach unten eine auffallende, fast gleichmäßige Zunahme des Kalifixiervermögens, das einerseits auf die Ablagerung eines ehemals verwitterten Flyschmaterials und andererseits auf eine langandauernde Verwitterung seit seiner Sedimentation in situ hinweist. Da kein Horizont besonders hervorspringt, liegt auch keine autochthone, fossile

3) freundl. schriftliche Mitteilung vom 12. 4. 1971. 
Bodenbildung vor. Nach oben wird dieses umgelagerte Bodenmaterial immer mehr mit Löß vermischt, wie dies auch aus dem zunehmenden Karbongehalt ersichtlich ist." Die mir von JANIK übersandten Analysenwerte sind im Anhang zu dem Aufsatz enthalten. Die Feststellung, daß das untersuchte Substrat nach obenhin stärker mit Löß (karbonatreich) durchsetzt ist, scheint mir trivial. Der genetische Mechanismus einer Durchmischung bis $\mathrm{zu}$ einer Tiefe von $2 \mathrm{~m}$ ist durch die Feststellung nicht geklärt. Ich halte das Sedimentpaket, das ich in der Aufschlußbeschreibung (Abb. 2) als Akkumulation IV bezeichne, für eine junge äolische Lößauflage auf z. T. äolischen und solifluidalen Staubsedimenten. Diese Deutung wird durch den außerordentlich niedrigen Strahlungspegel in $2 \mathrm{~m}$ Tiefe unterstützt. Ebenso ist an der Karbonatuntergrenze ein Ansteigen des Gehaltes an organischem Kohlenstoff zu beobachten. Ja selbst die von JaNiK übermittelten Austausch- und Fixierungswerte für $\mathrm{K}+$-Ionen zeigen an diesem Übergang in $2 \mathrm{~m}$ Tiefe einen Anstieg um $100 \%$. Eindeutigere Indizien für einen Sedimentationswechsel kann es m. E. nicht geben. Es bleibt die Frage offen: Handelt es sich bei den Horizonten mit org. C-Anreicherung um pleistozäne Landoberflächen? Erste Anhaltspunkte liefert die Interpretation der Redox-Verhältnisse innerhalb der unterschiedenen Sedimentationskörper.

\section{c) $\mathrm{Das} \mathrm{Fe} \mathrm{II}^{\mathrm{II}} \mathrm{Fe}$ III-Verhältnis}

Zur Indikation der Redoxverhältnisse im Prơfil scheint mir das Verhältnis von zweiwertigen zu dreiwertigem Eisen als geeignet. Es wurde im salzsauren Auszug nach Reinhard-Zimmermann bestimmt. Nach Gillespie (1920) werden die Reduktions-Oxidationsverhältnisse der Böden durch die in der organischen Substanz und im biologischen Zustand des Bodens eintretenden Veränderungen weitgehend beeinflußt. Aus Untersuchungen von Remezov (1930), die er auf unberührten Böden durchführte, geht hervor, daß der $\mathrm{r}_{\mathrm{H}}$-Wert (= negativer Logarithmus des im System auftretenden Wasserstoffgasdruckes $\mathrm{pH}_{2}$ ) in Böden bis zum C-Horizont abnimmt. Das Milieu im Solum ist reduzierender als im liegenden Substrat. In die gleiche Richtung weisen Arbeiten von Serdobolskij (1953), er stellt den großen Einfluß der Oberflächendurchfeuchtung auf die Verschiebung des Redoxpotentials im Boden heraus. Die von mir gefundenen Analysenwerte unterstützen diese Aussagen. Es ist geradezu erstaunlich, wie die Oxidationswerte von der Basis des jeweiligen Sedimentkörpers zu seiner Oberfläche hin abnehmen. Lediglich in dem Bereich des karbonatreichen Sediments verliert sich diese Regelhaftigkeit. Das ist ein erneuter Beweis dafür, daß der hangende Komplex ein lithomorph eigenständiges Gebilde darstellt, dessen Genese mit dem Liegenden wenig Gemeinsames hat. Interpretiert man die gefundenen Werte nach SerdobolskiJ, so lag der Sedimentationsbeginn der im Aufschluß enthaltenen Körper jeweils in einer ariden, das Sedimentationsende dagegen in einer humiden Phase des Pleistozäns. Ebenso müßte man annehmen, daß das Trockenmaximum zu Beginn der Akkumulation II (s. Aufschlußbeschreibung S. 3) lag und mit dem Ausklingen des Eiszeitalters zunehmend feuchtere Verhältnisse in dem Untersuchungsgebiet geherrscht haben. Dieses Ergebnis scheint mir hinreichend zu bestätigen, daß die Anreicherungshorizonte des organischen Kohlenstoffs fossile Reste von pleistozänen Böden darstellen. Um die an dieser Stelle schon deutlich erkennbare Systematik der Paläopedogenese weiter zu erhellen, soll im Folgenden die Diskussion weiterer im Labor gefundenen Ergebnisse dienen.

3) Physikalische Untersuchungen an den Proben

a) Der Retentionswert und sein Bezug zur Kornverteilung im Sediment

Bei dieser Methode handelt es sich um ein in der Chemie zur Trennung und der Identifikation von Substanzen häufig angewandtes Verfahren. Es beruht auf der unterschiedlichen Anlagerungsfreudigkeit der zu trennenden Substanzen an der Oberfläche eines 
Adsorbenten. Wir benutzen umgekehrt einen kationoiden Farbstoff zur Ermittlung der Adsorptionsaktivität verschiedener Böden (Metzger 1970). Nach Weinkötz (1972) unterscheiden sich die pedogenetischen Prozesse der Bodenbildung auf Schotterkörpern von jenen auf Löß durch die Anreicherung von Quarz im ersten und die von Tonmineralen im zweiten Fall in der Kornfraktion $\langle 20 \mu \mathrm{m}$.

Vergleicht man den Verlauf der $\mathrm{R}_{\mathbf{f}}$-Werte mit dem des Gehalts an Material der Kornfraktion unter $20 \mu \mathrm{m}$ der entsprechenden Proben (Abb. 5), so läßt sich im allgemeinen eine Übereinstimmung mit den Befunden von WeINкötz ablesen. In Analogie zur Aus-

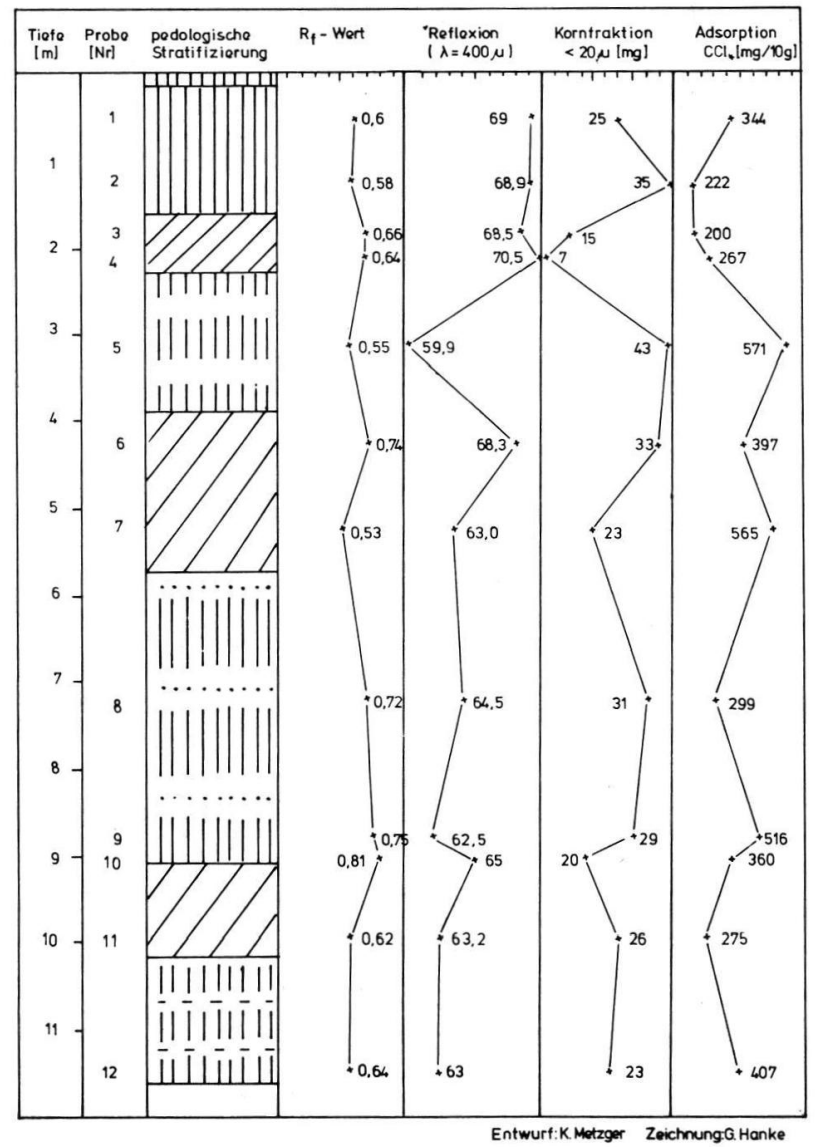

Abb. 5. Die aus physikalischen Meßwerten abgeleitete pedologische Stratifizierung. The pedological order found from physical analysis data.

wertung der Gamma-Kurve lassen sich die $\mathrm{R}_{\mathrm{f}}$-Werte in drei Gruppen einordnen. Im Akkumulationskörper II überwiegen die Werte $>0,70$ in den Körpern I und III + IV jene Werte $\left\langle 0,70\right.$. Der Verlauf der $R_{f}$-Werte zeigt eine abnehmende Adsorptionstendenz für kationoide Moleküle von der Basis der Sedimentpakete zu ihrer Oberfläche hin. Hierbei trägt mit Sicherheit der Eluvierungsgrad der vorliegenden Paläosolreste bei. Daraus schließe ich, daß durch die Perkolation eine Verfrachtung von Tonmineralen aus den noch erhaltenen Fragmenten der heute relikten oder fossilen $\mathrm{B}_{\mathrm{t}}$ - und $\mathrm{B}_{\mathrm{v}}$-Horizonte in das liegende Substrat erfolgte. Dieser Vorgang wurde wesentlich durch die mit der auslaufenden 
Kaltzeit einsetzende Perstrukturierung gefördert. Nach Kopp (1970) ist die Perstrukturierung für die zerstörten Profile älterer quartärer Böden verantwortlich. Ein einleuchtender Grund auch dafür, daß bodentypologische Untersuchungen mittel-, alt- und ältestpleistozäner Bodensedimente, wie sie von BRUNNACKER (1964 usw.) durchgeführt werden, sehr problematisch sind und kaum zur Grundlage einer quartären Bodenstratigraphie gemacht werden können. Diese Ansicht äußert auch FINK (1970), wenn er schreibt: „Eine genaue Alterseinstufung von Böden auf Grund ihrer typologischen Ausbildung ist sehr schwierig.“ Nach seinen Worten liegt die praktische Bedeutung der Paläopedologie darin, daß relikte Merkmale in Böden als solche erkannt und diese dann nicht der heutigen Dynamik zugeschrieben werden. Ein derartiges Merkmal ist beispielsweise die Farbe des Pedons, die sich doch in der Regel recht deutlich vom Substrat abhebt. Zu ihrer objektiven Erfassung diente die Messung der Remissionswerte.

\section{b) Das Reflexionsvermögen}

Die Untersuchungen werden von uns mit einer Apparatur der Fa. B. Lange, Berlin, die ursprünglich der Ermittlung des Weißgehaltes fester Oberflächen diente, durchgeführt. Böнm (1969) schreibt in seiner Arbeit, daß die Stärke des von einem Boden zurückgestreuten Lichtanteils von Parametern, wie Oberflächenform und Größe der Bodenteilchen, sowie vom Charakter des Materiales selbst beeinflußt wird. Er erkennt aus den an verschiedensten Bodensedimenten ermittelten Reflexionswerten eine systematische Sequenz die zu deren Datierung herangezogen werden könnte. In Abb. 5 sind die Reflexionswerte für den roten Anteil des sichtbaren Lichtes wiedergegeben. Unübersehbar ist auch in dieser Darstellung das Ansteigen der Werte in den Bereichen der vermuteten ehemaligen Landoberflächen. In Analogie zu den oben erwähnten Untersuchungsergebnissen von BöHM ist im vorliegenden Falle eine Abnahme der prozentualen Reflexion von 70,5\% für die angenommene Innerwürmoberfläche über $68,3 \%$ für die R/W-Oberfläche nach $65 \%$ für die M/R-Oberfläche zu beobachten. Nach diesen erneuten Hinweisen der Laborergebnisse auf pleistozäne Landoberflächen wage ich erstmals eine Zuordnung, wobei ich mir der Problematik wohl bewußt bin. Es handelt sich um das Nachvollziehen eingefahrener Vorstellungen. In diesem Falle jenen von Fink (1961a), der in seinem Exkursionsführer „Der östliche Teil des nördlichen Alpenvorlandes“ darauf hinweist, daß als locus typicus der fossilen Böden für die feuchte Lößlandschaft der Linzer Raum herangezogen wurde. Konkret bedeutet diese Feststellung die Zuordnung der in $4 \mathrm{~m}$ Tiefe beginnenden ehemaligen Landoberfläche zu der unter der Arbeitsbezeichnung „Linzer Komplex “ zusammengefaßten Bodenbildung der letzten Warmzeit und der Fließerden des darauffolgenden Frühglazials.

Bevor ich endgültig den Versuch unternehme, die durch chemische und physikalische Daten aufgezeigten Horizonte zu stratifizieren, sollen die röntgenographischen Mineralbestimmungen diskutiert und mit den bereits beschriebenen Aussagen verglichen werden.

\section{4) Mineralogische Untersuchung der Tonfraktion $\langle 2 \mu \mathrm{m}$}

Die im weiteren besprochenen Mineralbestimmungen sind auf Proben aus der Tonfraktion beschränkt. Dabei soll besonders das Verhältnis der verschiedenen Tonminerale untereinander, sowie jenes der Tonminerale zu Quarz herausgearbeitet werden.

U. Hofmann gab die Anleitung, nach der aus einer 0,1\%igen wäßrigen n-Butanol-(1)Suspension die Fraktion $\langle 2 \mu \mathrm{m}$ gewonnen wurde. Darauf folgte die übliche Vorbehandlung, um von den Präparaten Aufnahmen in der Debye-Scherrer-Kammer machen zu können. Die erhaltenen Negative wurden nicht wie üblich mit einem Maßstab vermessen, sondern unter einem Mikroskop ausgewertet, das zu diesem Zweck verändert wurde. Dazu 
habe ich das Okular eines Zeiss-Mikroskopes GFL/Pol durch einen Photowiderstand S 20 der Fa. Dr. Lange, Berlin, ersetzt, die Röntgenfilme zugeschnitten und zwischen Objektträgern fixiert. Die Auswertung der so erhaltenen Präparate erfolgte dadurch, daß man sie mit einer konstanten Geschwindigkeit (Synchronmotor und Getriebe) unter dem eingestellten Objektiv des Mikroskops durchführt. Der unterschiedliche Schwärzungsgrad des Films ändert die auf den Photowiderstand fallende Lichtstärke, die so hervorgerufenen Stromstärkeschwankungen werden durch einen synchron laufenden Linienschreiber aufgezeichnet. ${ }^{4}$ )

Als Vergleichspräparate zur Peakflächenermittlung dienten solche reiner Tonminerale, Quarz und primärer Silikate sowie deren Mischungen in den unterschiedlichsten Verhältnissen. Die quantitative Auswertung erfolgte durch den Vergleich der Schreiberdiagramme, dabei werden die Peakflächeninhalte der Basisinterferenzen einander gegenüber gestellt. Die Methode eignet sich besonders gut, um die Verhältnisse Quarz zu Tonmineralen in Böden und deren Substraten zu beschreiben. Brauchbar sind daneben auch die Werte, die Minerale der Kaolinitgruppe liefern. Dagegen ist die quantitative Auswertung der Dia-

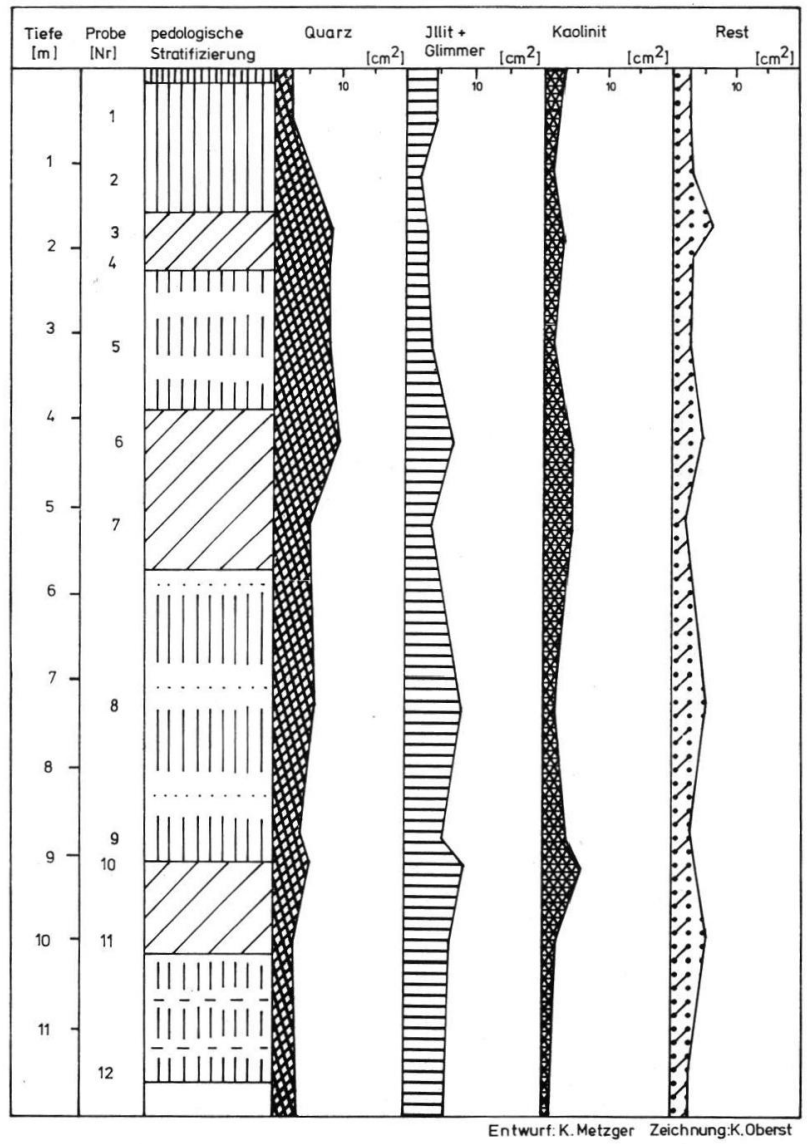

Abb. 6. Die pedologische Stratifizierung im Vergleich zum Gang der Tonminerale. The pedological order compared with the charakter of clay-minerals.

4) Bei der Analyse der Filme und Diagramme hat mir Herr Prof. U. Hofmann wichtige Ratschläge erteilt. Ich möchte mich dafür an dieser Stelle bedanken. 
gramme zur Ermittlung der Illit-, Chlorit, Vermiculit- und Montmorillonitverhältnisse nicht befriedigend. So wird beispielsweise die typische Interferenz des Vermiculits von solchen des Chlorits und Montmorillonits gestört. Das gleiche Problem tritt bei der Unterscheidung von Illit und primären Silikaten (Glimmer) auf. In diesem Fall führt die angewandte Methode zu keinen Ergebnissen. Verzichtet man jedoch auf die absolute Genauigkeit, dann lassen sich mit relativ geringem präparativem Aufwand halbquantitative Aussagen machen,

a) über den Gang des Quarzgehaltes

b) des Glimmer und Illitgehaltes

c) des Gehaltes an Mineralien der Kaolinitgruppe und

d) der Resttonminerale wie Montmorillonit, Chlorit und Vermiculit.

Die genauesten Werte erhält man vom Quarz, selbst dann, wenn verhältnismäßig viel Glimmer und Illit neben Quarz vorliegt. Die Minerale der Kaolinitgruppe werden zusammengefaßt und anhand ihrer charakteristischen Basisinterferenzen der 1. und 2. Ordnung ausgewertet. Auf eine Unterscheidung zwischen der Illit- und Glimmergruppe muß wegen der dabei auftretenden Schwierigkeit verzichtet werden. Zur Auswertung wird die für die Minerale typische Basisinterferenz der 1 . Ordnung bei $d=10,0$ herangezogen. Im Rest schließlich sind Minerale der Chloritgruppe, Montmorillonit und Vermiculit zusammengefaßt, deren charakteristische Interferenzen um $d=14$ und darüber liegen. Die aus den jeweiligen Peaks ausgemessenen Werte sind in Abb. 6 wiedergegeben. Zur sachlichen Klärung möchte ich bemerken, daß es sich dabei nicht um absolute Werte handeln kann. So wurde beispielsweise die stärkste Quarzlinie bei $\mathrm{d}=3,33$ herangezogen, die bekanntlich mit Interferenzen von Illit und Glimmer koinzidiert. Es ist also nicht ausgeschlossen, daß die Quarzwerte insgesamt zu hoch liegen. Die gleiche Überlegung gilt auch für die übrigen Gruppen.

Rein qualitativ kann aufgrund der Ergebnisse ausgesagt werden:

a) Der Quarzgehalt nimmt im Aufschluß zu der Basis hin ab, dazu gegenläufig ist das Verhältnis von Illit und Glimmer.

b) Der Kaolinitgehalt verläuft im allgemeinen recht gleichmäßig mit zwei ausgesprochenen Maxima der Probe 6, aus $4 \mathrm{~m}$ Tiefe und Probe 10 aus 9,10 m Tiefe.

c) Der als Rest zusammengefaßte Anteil der gemeinsam erfaßten Tonminerale zeigt neben einer sonst gleichmäßigen Verteilung ebenfalls Maxima bei den Proben 6, 8 aus 7,25 m Tiefe und der Probe 10.

d) Die unter c) angesprochenen Anhäufungen zeigen auch die Verteilungskurven von Quarz und Illit/Glimmer.

Es ist nicht ganz einfach, das tonmineralogische Untersuchungsergebnis im Zusammenhang mit den physikalisch-chemischen Fakten sowie mit der im Aufschlußbefund wiedergegebenen Situation zu interpretieren.

Entscheidend sind meiner Ansicht nach die Konzentrationen von Kaolinit.

Nach dem heutigen Stand der Tonmineralforschung ist Kaolinit ein typisches Mineral der kontinentalen Verwitterung, die durch perkolierende Lösungen hervorgerufen wird. Daher halte ich die beiden im Aufschluß auftretenden Maxima für Zeugen ehemaliger Landoberflächen, die einer intensiven Verwitterung unterlagen. Es ist sicherlich nicht falsch, wenn man den im Substrat vorhandenen Kaolinitgehalt einer ehemaligen der Deflation ausgesetzten Sedimentoberfläche zuschreibt. Glass, PotTer \& Siever (1956) sowie Смоот (1960) haben durch ihre Untersuchungen an Geröll- und Geschiebeoberflächen ge- 
zeigt, daß die sandigen Schichten meist einen höheren Kaolinitgehalt aufweisen als die tonigen. Darüber hinaus wird selbst die von Millot (1949) gefundene $\mathrm{PH}$-Abhängigkeit in bezug auf Kaolinitanreicherungen im Sediment bestätigt. BAUER (1972) hat in den Bodensuspensionen mit destilliertem Wasser nach 24 Stunden $\mathrm{p}^{\mathrm{H}}$-Werte $\mathrm{zwischen}$ 6,7 und 5,8 ermittelt.

Im Gegensatz zur Diagenese des $Z_{w}$ eischichtminerals Kaolinit läßt sich die der Dreischichtminerale, Illit, Montmorillonit und Chlorit nicht so eindeutig erklären. Zunächst findet sich der von SALGER (1954) festgestellte Gang des Quarz- und Glimmer-/Illitgehaltes bei der Verwitterung und Bodenbildung auf diluvialen Schottern auch bei den hier untersuchten Lößsedimenten wieder. Der Quarzgehalt nimmt mit dem Alter des Sediments deutlich ab. Der Gesamttongehalt liegt aber in seinem Maxima weit über den bei SALger angegebenen Werten.

Das ist ein Phänomen, das von WeINkötz (1972) durch $\mathrm{R}_{\mathbf{f}}$-Wertuntersuchungen an Lößsedimenten des Kaiserstuhls gefunden und gedeutet wurde. Die Neubildung von Dreischichttonmineralen findet in der Natur nur in alkalischer und kationreicher Umgebung statt (Millot 1970). Für unseren Aufschluß würde das bedeuten, daß sie schon überwiegend im Sediment vorhanden waren. Die Anreicherung in bestimmten Horizonten des Aufschlusses ist somit eine Funktion ihrer Verwitterungsstabilität im Gegensatz zu anderen anorganischen Bestandteilen des Sediments. Ein weiterer Beweis dafür, daß Anreicherungsschichten im Aufschluß ehemalige Landoberflächen waren. Ebenso läßt sich die Abnahme dieser Tonminerale zur heutigen Oberfläche hin als Verdünnungseffekt deuten, so daß ihre Genese im Alt- und Altestpleistozän gelegen hat. Diese Hypothese stützt die Annahme, daß dort in den Interglazialen ein wesentlich wärmeres und feuchteres Klima geherrscht haben muß. BLüthgen (1964) meint, daß es anhand von C-14 und O-18 Bestimmungen möglich ist, für das gesamte Pleistozän eine absolute Zeitskala der Temperaturschwankungen aufzustellen (S. 559). Die dort beschriebenen Ergebnisse kommen den von mir entwickelten Vorstellungen nahe.

\section{Auswertung}

Die Temperatur des Karibischen Meeres war im Würm I/II-Interstadial um $4^{\circ} \mathrm{C}$ niedriger $\left(24^{\circ} \mathrm{C}\right)$ als heute $\left(28^{\circ} \mathrm{C}\right)$, das läßt sich aus dem bei BLÜTHGEN wiedergegebenen Diagramm entnehmen. Das bedeutet, daß das Julimittel der Temperatur im Welser Raum bei ca. $10^{\circ} \mathrm{C}$ lag. Unter diesen Bedingungen kann die Bodenentwicklung im Würm I/IIInterstadial nicht über ein Tundra-Naßgleystadium heutiger borealer Klimaprovinzen hinausgegangen sein. Diese Erkenntnis wird durch Untersuchungen der Lößabfolgen in Württemberg, Sachsen, Bayern, Nieder- und Oberösterreich von WEIDENBACH (1953), Freising (1954), Lieberoth (1962, 63), Brunnacker (1957, 1960), Felgenbauer, Fink \& De VRIEs (1959), Fink (1961a, 1962, 1964) bestätigt. Auch die in dieser Arbeit wiedergegebenen Gelände- und Laborbefunde weisen eindeutig auf Bildungsbedingungen für die vorletzte "Landoberfläche“ hin, die mit Sicherheit von denen der im Liegenden vorhandenen abweichen. Aus dem höheren Reduktionspotential, dem relativ geringen Anteil von org. Kohlenstoff und dem niederen Adsorptionsvermögen gegenüber unpolaren Lösungsmitteln schließe ich auf eine im kühl-humiden Klimabereich abgelaufene Bodenentwicklung, die mit Stillfried B gleichgesetzt werden kann. Die im Liegenden in $4 \mathrm{~m} \mathrm{u}$. Ok. auftretende Zersatzzone besitzt dagegen ein wesentlich höheres Reduktionspotential und kommt durch auffällige Anreicherung von Tonmineralen und organischem Kohlenstoff zum Vorschein. Eine durch diese Parameter gekennzeichnete Verwitterungsintensität kann unmöglich unter den für die hangende Oberfläche geltenden Klimabedingungen erfolgt sein. Damit ist diese fossile Landoberfläche dem bei FINK (1961a) beschriebenen „Linzer 
Komplex“ d.h. dem Stillfrieder Komplex gleichzusetzen. Die von mir gefundenen und interpretierten Werte unterstreichen die Feststellung von Moll (1970), daß die Entwicklung eines rubefizierten Lößverwitterungsbodens nur unter einem wesentlich wärmeren und feuchteren Klima als heute entstanden sein kann. Die gleiche Auffassung vertritt auch Graul (1962), der sich sehr eingehend mit den Interpretationen von WitTManN und MAROCKe auseinandergesetzt hat. Die beiden Autoren hatten die Parallelisierung der rubefizierten Schotterböden auf der älteren Teilterrasse des Oberrheintals mit dem Göttweiger Boden vorgenommen und dem Würm I/II-Interstadial zugeordnet. Inzwischen dürften die Befunde ausreichen, daß die Zuordnung dieser Bodenbildungen der Eem-Zeit, d. h. dem Riß/Würm-Interglazial als gesichert gelten kann.

Die älteste im vorliegenden Aufschluß hervortretende fossile Oberfläche ist durch auffallende Extremwerte gekennzeichnet. Im Gegensatz zu der im Szintillationsdiagramm kaum sichtbaren Riß/Würm-Oberfläche tritt diese ältere Verwitterungsbildung äußerst deutlich auf. Am auffallendsten ist das Ansteigen des Kohlenstoffgehalts und das Absinken des Oxidationspotentials, ein eindeutiges Zeichen für extreme Bedingungen zur Zeit der Oberflächenbildung. Hier ist nach dem Gesagten ausgeschlossen, daß es sich um eine interstadiale Oberfläche handelt. Wenn im Hangenden kein Hiatus auftritt, kann diese in $9 \mathrm{~m}$ Tiefe auftretende Diskontinuitätsfläche als ehemalige Mindel/Riß-Oberfläche angesehen werden. Mißt man dem Szintillationsdiagramm einen gewissen Aussagewert bei, so läßt sich die Einheitlichkeit des hangenden Komplexes schon am Kurvenverlauf (Abb. 2) feststellen. Das Ergebnis würde damit lauten: „Der liegende Schotter bei Wels ist mindestens eine günzeiszeitliche Ablagerung. “ Zu diesem Ergebnis gelangt auch JANIK (1971) ${ }^{5}$ ), wenn er schreibt: „Der liegende Lehm wurde vom Aiterbach, einem Nebengerinne der Traun, im Altpleistozän abgelagert, als der Krems-Steyr-Gletscher seine Moränen bei Ried im Traunkreis (südl. Kremsmünster) aufschüttete. "Die heutige Höhenlage des Profils begründet er mit einer Landhebung und einer dadurch bedingten Einschneidung der Traun. Dieses Postulat ist durch die von mir vorgenommenen Untersuchungen weder bestätigt noch widerlegt. Dagegen ist die Annahme von JANIK, daß die von ihm durchgeführten makro- und mikromorphologischen Untersuchungen sowie die chemischen Analysen keine fossile Oberfläche erkennen lassen, irrig. Wie ich gezeigt habe, treten ganz im Gegenteil mit recht großer Sicherheit zwei fossile Interglazialoberflächen und eine Interstadialoberfläche im Profil deutlich hervor. Es handelt sich nicht um einen einheitlich akkumulierten Sedimentkörper, vielmehr um mindestens vier deutlich voneinander unterscheidbare $\mathrm{Ab}$ lagerungen, wobei man die drei älteren Sedimente durchaus als fluvioglaziale Ablagerungen ansprechen konnte. Es fehlen teilweise die mikro- und makromorphologischen Symptome, die man einem äolischen Sediment zuschreibt. Mir scheint, als ob die Problematik der Genese derartiger Lockersedimente sehr eng mit der regionalen Unterscheidung in trockene und feuchte Lößlandschaften, wie sie von FINK vorgenommen wurde, zusammenhängt. Man findet ähnlich gelagerte Fälle auch in der südlichen Hälfte des Oberrheintalgrabens sowie in den Gebieten der ehemaligen Rhein- und Iller/Lech-Gletscher wieder. Dagegen zeigen die Ziegeleiaufschlüsse im Raum Niederbayern, deren Liegendes aus tertiären Schottern oder Sanden gebildet wird, den Habitus der Lösse feuchter Landschaften. In der chemischen Analyse zeichnet sich dieser Typ durch äußerst niedrige Kohlensäurewerte aus.

In dem hier beschriebenen Profil müssen bei der Ablagerung des jüngsten SubstratKomplexes andere klimatische Verhältnisse geherrscht haben, als dies bei jener der älteren Schichten der Fall war. Der ansteigende Karbongehalt deutet auf ein trockenes Klima hin, indem die vom Wind verfrachteten Teilchen nicht gleichzeitig einer Verspülung durch die „hohen Niederschläge“ unterlagen.

5) freundl. schriftliche Mitteilung vom 12. 4. 1971. 
Das vorliegende Profil zeigt auf dem liegenden günzeiszeitlichen Schotter eine als Akkumulation I bezeichnete mindeleiszeitliche Lößlehmauflage. Dieser Lehm ist nach oben hin deutlich verwittert und schließt auch im Aufschluß sichtbar mit einer Diskontinuitätsfläche ab. Das darauf folgende $5 \mathrm{~m}$ mächtige Lehmpaket wird als Akkumulation II bezeichnet und der Rißeiszeit zugeordnet. Die Verwitterungszonen sind im Aufschluß nicht zu erkennen, sie treten aber bei der Laboruntersuchung von Probematerial, deutlich heraus und bilden den Abschluß zur Akkumulation III, einem Lößlehm, der sich deutlich von seinem Liegenden absetzt und dem Würm I zugerechnet wird. Die zwischen diesem und dem darauffolgenden kalkhaltigem Löß der Akkumulation IV auftretende Zersatzzone stellt nach den ausgeführten Überlegungen eine interstadiale Oberfläche zwischen Würm I und II dar.

Analysenergebnisseder Ziegelei Würzburger, Wels-Thalheim (n. V. JANIK)

\begin{tabular}{|c|c|c|c|c|c|c|c|c|}
\hline Nr. & Tiefe & ${ }_{\mathrm{KC}}^{\mathrm{PH}}$ & ${ }_{\mathrm{Az}}^{\mathrm{pH}}$ & $\begin{array}{c}\mathrm{P}_{2} \mathrm{O}_{5} \\
\mathrm{mg} / 100 \mathrm{~g}\end{array}$ & $\begin{array}{l}\mathrm{K}_{2} \mathrm{O} \\
\text { Boden }\end{array}$ & $\mathrm{CaCO}_{3} \%$ & $\begin{array}{l}\mathrm{K} \\
\text { aust. } \\
\mathrm{mg} \text { / }\end{array}$ & $\begin{array}{c}\mathrm{K}_{\mathrm{fix}} . \\
100 \mathrm{~g}\end{array}$ \\
\hline 1 & $0,6 \mathrm{~m}$ & 6,8 & 7,2 & 2 & 8 & 13,4 & 7.15 & 27,25 \\
\hline 2 & $1,3 \mathrm{~m}$ & 6,9 & 7,3 & 1 & 7 & 16,8 & 5,87 & 23,27 \\
\hline 3 & $1,75 \mathrm{~m}$ & 6,7 & 7,2 & 2 & 7 & 1,7 & 5,00 & 37,50 \\
\hline 4 & $2,10 \mathrm{~m}$ & 6,5 & 7,1 & 2 & 6 & 0,6 & 5,00 & 35,00 \\
\hline 5 & $3,00 \mathrm{~m}$ & 5,7 & 6,9 & 2 & 13 & 0,8 & 12,00 & 44,50 \\
\hline 6 & $4,00 \mathrm{~m}$ & 5,8 & 7,0 & 3 & 9 & 0,6 & 7,12 & 54,62 \\
\hline 7 & $5,25 \mathrm{~m}$ & 5,9 & 7,0 & 1 & 4 & 0,2 & 4,50 & 69,50 \\
\hline 8 & $7,25 \mathrm{~m}$ & 6,0 & 7,0 & 3 & 4 & - & 4,00 & 64,00 \\
\hline 9 & $8,8 \mathrm{~m}$ & 5,9 & 7,1 & 3 & 8 & 0,4 & 6,75 & 66,75 \\
\hline 10 & $9,10 \mathrm{~m}$ & 6,0 & 7,0 & 3 & 9 & 0,6 & 3,37 & 60,87 \\
\hline 11 & $11,00 \mathrm{~m}$ & 6,0 & 7,1 & 3 & 8 & 0,4 & 4,87 & 64,87 \\
\hline 12 & $11,90 \mathrm{~m}$ & 6,1 & 7,0 & 6 & 4 & 0,4 & 6,75 & 41,75 \\
\hline
\end{tabular}

Liter a t u r

BAUER, A.: Laboruntersuchungen bogengenetischer Prozesse auf Lößsedimenten im Raum Linz/ Oberösterreich. - Staatsexamensarbeit Geograph. Inst. Univ. Heidelberg 1971.

Böнм, W.: Altersuntersuchungen an fossilen und relikten Böden auf Quartärschottern Oberösterreichs. - Staatsexamensarbeit Geograph. Inst. Univ. Heidelberg 1969.

BlÜthGEN, J.: Allgemeine Klimageographie. - 2. Auflage (Lehrbuch der Allgemeinen Geographie, Band II), Berlin 1966.

BrunNaCker, K.: Die Geschichte der Böden im jüngeren Pleistozän in Bayern. - Geologica Bavarica 34, 1-95, 11 Abb., 3 Tab., 2 Taf. München 1957.

-- : Zur Kenntnis des Spät- und Postglazials in Bayern. - Geologica Bavarica 43, 74-150, 13 Abb., 16 Tab., München 1959.

- : Grundzüge einer quartären Bodenstratigraphie in Süddeutschland. - Eiszeitalter u. Gegenwart 15, 224-228, Öhringen 1964.

Felgenbauer, J., Fink, J. \& DE Vries, H.: Studien zur absoluten und relativen Chronologie der fossilen Böden in Österreich. - Archäologica Austriaca 25, Wien 1959.

Fezer, F.: Tiefenverwitterung circumalpiner Pleistozänschotter. - Heidelberger Geograph. Arb. 24, 144 S., 90 Abb., 2 Taf., Heidelberg 1969.

Fink, J.: Zur Korrelation der Terrassen und Lösse in Österreich. - Eiszeitalter u. Gegenwart 7, 49-77, 9 Abb., OHhringen 1956.

- : Der östliche Teil des nördlichen Alpenvorlandes. - Mitt. Österr. Bodenkundl. Ges. 26, H. 6, 26-51, 9 Abb., Wien 1961 (1961 a).

- : Die Gliederung des Jungpleistozäns in Ơsterreich. - Mitt. geol. Ges. Wien 54, 1-25, 1 Taf., Wien 1962 (1962 a).

— : Die Böden Niederösterreichs. - Jb. Landesk. von Niederösterreich 36, 965-984, Wien 1964.

- : Grenzen der Paläopedologie. - Z. f. Pflanzenern. u. Bodenk. 121, 19-33, Weinheim 1970.

Fink, J. \& Franz, H.: Exkursionen durch Ớsterreich. - Mitt. Ósterr. Bodenkundl. Ges. H. 6, Wien 1961 (1961 b).

FrEISING, H.: Neue Ergebnisse der Lößforschung im nördl. Württemberg. - Jb. geol. Abt. Württemb. Stat. Landesamt, 1, 54-59, Stuttgart 1951. 
Gillespie, K. G.: Soil Sience, 9, 199, Baltimore 1920.

Blass, H. D., Potter, P. E. \& Siever, R.: Clay mineralogy of some basal Pennsylvanian sandstones, clays and shales. - Bull. Am. Ass. Petrol. Geol. 40, 750-754, 4 Abb., Tulsa 1956.

Graul, H.: Untersuchungen über Abtragung und Aufschüttung im Gebiet des unteren Inn und des Hausruck. — Mitt. Geogr. Ges. München 30, 180-259, München 1937.

- : Geomorphologische Studien zum Jungquartär des nördl. Alpenvorlandes. Teil I. Das Schweizer Mittelland. - Heidelberger Geograph. Arb. 9, Teil 1, 104 S., 6 Fig., 6 Tafeln, Heidelberg 1962 (1962 a).

- : Die Niederterrassenfelder im Umkreis von Basel. - Eiszeitalter u. Gegenwart 13, 181-196, 3 Abb., Ohringen 1962 (1962 b).

- : Eine Revision der pleistozänen Stratigraphie des schwäbischen Alpenvorlandes. - Peterm. Geogr. Mitt. Jg. 106, 4c, 253-271, Gotha 1962 (1962 c).

Hofmann, U.: Aus der Chemie des Tons. - Angew. Chem. 80, Nr. 18, 736-747, Weinheim 1968.

JANIK, V.: Die Bodenentwicklung auf der Hochterrasse der Traun bei Linz. - Naturkundl. Jb. d. Stadt Linz, 39-105, 13 Diagr., 7 Tab., 2 Karten, 13 Bilder, Linz 1965.

- : Die Genetik der Sedimente auf der Traunhochterrasse bei Linz. - Naturkundl. Jb. der Stadt Linz, 7-121, 1 Karte, 8 Tab., 23 Diagr., 4 Bilder, Linz 1967.

- : Die Linzer Lößprofile in pedologischer und epirogen-tektonischer Sicht. - Naturkundl. Jb., der Stadt Linz, 235-255, 6 Tab., 2 Taf., 8 Bilder, Linz 1969.

Koнl, H.: Die Exkursion zwischen Lambach und Enns. - Verh. Geolog. B. A., Sonderheft D, 40-62, Wien 1955.

Kopecky, J.: Methode zur Korngrößenbestimmung. — Int. Mitt. Bodenkunde, 4, 138-109, Berlin 1914.

Kopp, A.: Periglaziäre Umlagerungs-(Perstruktions-)zonen im nordmitteleuropäischen Tiefland und ihre bodengenetische Bedeutung. Beiträge zur Bodensystematik. - Int. bodenk. Symposium, DDR. Tagungsbericht Nr. 102, 55-81, 12 Abb., Berlin 1970.

KunN, E.: Neueste und verbesserte physik.-chemische Untersuchungsmethoden an Böden aus dem nördl. Bereich des alten Rheingletschers. - Staatsexamen, Geograph. Inst. Univ. Heidelberg 1971.

Lieberoth, J.: Die jungpleistozänen Lösse Sachsens im Vergleich zu denen anderer Gebiete. Petermanns Geogr. Mitt. 106, 3, 188-198, Gotha 1962.

- : Lößsedimentation und Bodenbildung während des Pleistozäns in Sachsen. - Geologie 12, 149-187, 13 Abb., 6 Tab., Berlin 1963.

Marocke, R.: Etude pédologique préliminaire des sols rouges et gris de Hardt. - Ann. Agron. 1956, 889-893, Paris 1956.

Metzger, K.: Physikalisch-chemische Untersuchungen an relikten und fossilen Böden im Nordgebiet des alten Rheingletschers. - Heidelberger Geogr. Arb., 19, 99 S., 21 Abb., 9 Tab., Heidelberg 1968.

- : Die Anwendung physikalisch-chemischer Meßmethoden zur Relativdatierung von fossilen und relikten Paläoböden. - Heidelberger Geogr. Arb. 38, 1-20, 2 Tab., Heidelberg 1973.

Millor, G.: Relations entre la constitution et la genèse des roches sédimentaires argileuses. Thèse Sci. Nancy et Géol. Appl. Prospec. Min. 2, uos 2, 3, 4, S. 1-352, Nancy 1949.

- : Geology of Clays. 438 S., 85 Abb. - New York, Heidelberg, Berlin (Springer) 1970.

Mǘckenhausen, E.: Die wichtigsten Bden der Bundesrepublik Deutschland. - 2. Aufl., Frankfurt M. 1959.

Remezov, N. P.: Proceedings 2nd Int. Congr. Soil Science. - Moskau - Leningrad 1930.

SALGER, M.: Verwitterung und Bodenbildung auf diluvialen Schotterterrassen. - Heidelberger Beiträge zur Mineralogie und Petrographie 4, 288-318, 7 Abb., Heidelberg 1954.

Serdobolskiy, I. P.: Bodenkunde, Bd. 1, 25, Moskau 1953 russ.

Sмоот, R. W.: Clay mineralogy of pre-Pennsylvanian sandstones and shales of the Illinois Basin, Part. I - Relation of permeability to clay mineral suites. - Illinois State Geol. Surv. 286, 20 S., 1960.

Urban, H.: Röntgenkartei zur Bestimmung von Ton- und Sedimentmineralien. - Opuscula Mineralogica et Geologica 5, 41 S., zahlr. Taf., Kettwig (Feller - Nottuln) 1954.

Weidenbach, F.: Zeitliche Einordnung der jungpleistozänen Ablagerungen Mitteleuropas. Actes IV. Congr. Intern. Quaternaire, 1-7, Rom 1953.

Weinkötz, I.: Versuch einer Stratifizierung der Lößablagerungen bei Bötzingen im Kaiserstuhl anhand von Laboruntersuchungen. - Staatsexamensarbeit Geogr. Inst. Univ. Heidelberg 1972.

Wittmann, O.: Die Niederterrassenfelder im Umkreis von Basel und ihre kartographische Darstellung. - Basler Beiträge Geogr. Ethnol. 3, 1-46, Basel 1961.

Manuskript eingeg. 9. 5. 1973.

Anschrift des Verf.: Dr. Kurt Metzger, Geomorphologisches Labor des Geographischen Instituts der Universität Heidelberg, 6900 Heidelberg 1, Berliner Straße 29. 